\title{
Pd(0)-Catalyzed PMHS Reductions of Aromatic Acid Chlorides to Aldehydes
}

Kyoungsoo Lee and Robert E. Maleczka, Jr.*

Department of Chemistry, Michigan State University, East Lansing, Michigan 48824

maleczka@chemistry.msu.edu

\section{Supporting Information}

Index

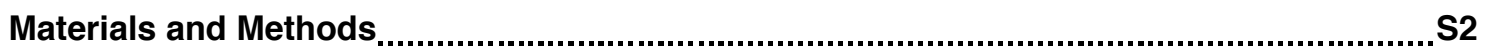

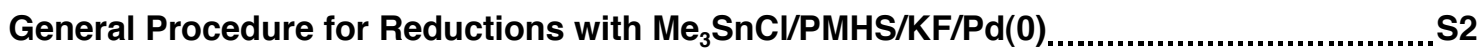

General Procedure for Reductions with PMHS/KF/Pd(0) ........................................S2-S3

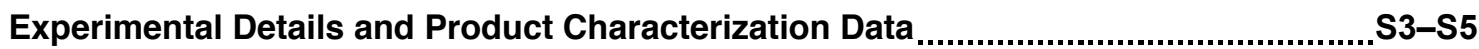

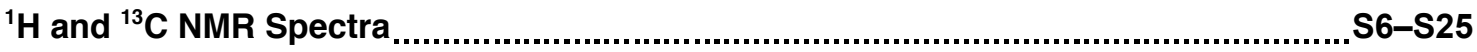


Materials and Methods: All reactions were carried out with magnetic stirring, and monitored by thin-layer chromatography with $0.25-\mathrm{mm}$ precoated silica gel plates or capillary GC with a fused silica column, unless otherwise noted. THF was freshly distilled from sodium/benzophenone under nitrogen. Tris(dibenzylideneacetone)dipalladium (0), tri-2-furylphosphine, anhydrous A.C.S grade potassium fluoride, polymethylhydrosiloxane (PMHS), and tetrabutylammonium fluoride (1M solution in THF) were purchased and used without purification unless otherwise mentioned. 2-Thiophenecarbonyl chloride, ${ }^{1}$ and 3,4,5-trimethoxybenzoyl chloride ${ }^{2}$ were prepared following literature procedures and used after distillation. The remaining acid chlorides were purchased and used without purification. Flash chromatography was performed with silica gel $60 \AA$ (230-400 mesh). Yields refer to chromatographically and spectroscopically pure compounds unless otherwise stated. ${ }^{1} \mathrm{H}$ NMR and ${ }^{13} \mathrm{C}$ NMR spectra were recorded on a $300 \mathrm{MHz}$ spectrometer (300 MHz for ${ }^{1} \mathrm{H}$, respectively, and $75 \mathrm{MHz}$ for ${ }^{13} \mathrm{C}$, respectively) with chemical shifts reported relative to the residue peaks of solvent chloroform $\left(\delta 7.24\right.$ for ${ }^{1} \mathrm{H}$ and $\delta 77.0$ for $\left.{ }^{13} \mathrm{C}\right)$. Melting points were measured on a capillary melting point apparatus and are uncorrected.

General Procedure for Reductions with $\mathrm{Me}_{3} \mathrm{SnCl} / \mathrm{PMHS} / \mathrm{KF} / \mathrm{Pd}(0)$ : $\mathrm{Pd}_{2} \mathrm{dba}_{3}(0.01 \mathrm{mmol}, 9.2$ $\mathrm{mg}$ ) and trifurylphosphine (TFP) $(0.04 \mathrm{mmol}, 9.3 \mathrm{mg})$ were added to THF $(5 \mathrm{~mL})$ and the resulting mixture was stirred at $\mathrm{rt}$ for $15 \mathrm{~min}$. At that time, acid chloride (1 mmol), $\mathrm{Me}_{3} \mathrm{SnCl}(0.1-1.0 \mathrm{mmol})$, PMHS (1.5 mmol, $0.09 \mathrm{~mL})$, aq KF (1.5 mmol, $87.3 \mathrm{mg}$ in $1 \mathrm{~mL} \mathrm{H}_{2} \mathrm{O}$ ) and TBAF (1 drop of a $1 \mathrm{M}$ solution in THF ( 0.008 mmol)) were added successively. The reaction was then allowed to stir at rt until the reduction was judged complete by GC ( $\sim .5$ to $1 \mathrm{~h})$. At that time, the reaction was diluted with saturated aq KF $(3 \mathrm{~mL})$ and stirred for $0.5 \mathrm{~h}$. The reaction was then extracted with $\mathrm{Et}_{2} \mathrm{O}$ and the aqueous phase was back extracted with $\mathrm{Et}_{2} \mathrm{O}$. The combined organics were dried over $\mathrm{MgSO}_{4}$, filtered, and concentrated. The resulting residue was purified by silica gel chromatography $\left(10 \% \mathrm{Et}_{2} \mathrm{O} /\right.$ pentane) to afford the aldehyde.

General Procedure for Reductions with PMHS/KF/Pd(0): $\mathrm{Pd}_{2} \mathrm{dba}_{3}(0.01 \mathrm{mmol}, 9.2 \mathrm{mg})$ and trifurylphosphine (TFP) $(0.04 \mathrm{mmol}, 9.3 \mathrm{mg})$ were added to THF $(5 \mathrm{~mL})$ and the resulting mixture was stirred at $\mathrm{rt}$ for $15 \mathrm{~min}$. At that time, acid chloride $(1 \mathrm{mmol})$, PMHS $(3.0 \mathrm{mmol}, 0.18 \mathrm{~mL})$, aq. $\mathrm{KF}\left(3.0 \mathrm{mmol}, 174.5 \mathrm{mg}\right.$ in $\left.1 \mathrm{~mL} \mathrm{H}_{2} \mathrm{O}\right)$ and TBAF (1 drop of a $1 \mathrm{M}$ solution in THF ( 0.008 mmol)) were added successively. The reaction was then allowed to stir at $r$ until the reduction was complete by $\mathrm{GC}$ monitoring $(\sim 1 \mathrm{~h})$. At that time, the reaction was extracted with $\mathrm{Et}_{2} \mathrm{O}$ and the aqueous phase back extracted with $\mathrm{Et}_{2} \mathrm{O}$. The combined organics were dried over $\mathrm{MgSO}_{4}$,

(1) DeVries, V. G.; Bloom, J. D.; Dutia, M. D.; Katocs, A. S., Jr.; and Largis, E. E. J. Med. Chem. 1989, 32, 2318-2325.

(2) Azzena, U.; Denurra, T.; Melloni, G.; and Rassu, G. J. Chem. Soc., Chem. Commun. 1987, 1549-1550. 
filtered, and concentrated. The resulting residue was purified by silica gel chromatography $(10 \%$ $\mathrm{Et}_{2} \mathrm{O} /$ pentane) to afford the aldehyde.

\section{Experimental Details and Spectroscopic Data:}

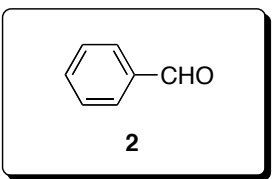

Benzaldehyde (Table 2, entry 1): Subjection of benzoyl chloride (1) (1.0 $\mathrm{mmol}, 0.141 \mathrm{mg}$ ) to the general procedure afforded after column chromatography (silica gel, $10 \% \quad \mathrm{Et}_{2} \mathrm{O} /$ pentane) $0.105 \mathrm{~g}(99 \%)$ of benzaldehyde (2) as a colorless liquid. ${ }^{1} \mathrm{H}$ NMR $\left(300 \mathrm{MHz}, \mathrm{CDCl}_{3}\right): \delta 9.98(\mathrm{~s}, 1 \mathrm{H}), 7.85$ (dd, $J=$ 8.0, 1.1 Hz, 2H), $7.62(\mathrm{~m}, 1 \mathrm{H}), 7.51(\mathrm{~m}, 2 \mathrm{H}) ;{ }^{13} \mathrm{C} \mathrm{NMR}\left(75 \mathrm{MHz}, \mathrm{CDCl}_{3}\right): \delta 192.2,136.3,134.3$, 129.6, 128.9. Physical and spectral data were consistent with those obtained from a commercial sample.

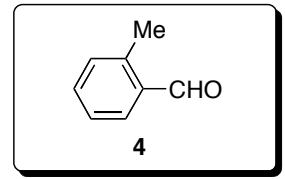

2-Methylbenzaldehyde (Table 2, entry 2): Subjection of 2-methyl-benzoyl chloride (3) $(1.0 \mathrm{mmol}, 0.155 \mathrm{mg})$ to the general procedure afforded after column chromatography (silica gel, $10 \% \mathrm{Et}_{2} \mathrm{O} /$ pentane) $0.100 \mathrm{~g}(83 \%)$ of 2methyl-benzaldehyde (4) as a colorless liquid. ${ }^{1} \mathrm{H} \mathrm{NMR}\left(300 \mathrm{MHz}, \mathrm{CDCl}_{3}\right): \delta$ $10.24(\mathrm{~s}, 1 \mathrm{H}), 7.78(\mathrm{~d}, J=7.4 \mathrm{~Hz}, 1 \mathrm{H}), 7.48(\mathrm{~m}, 1 \mathrm{H}), 7.35(\mathrm{~m}, 1 \mathrm{H}), 7.23(\mathrm{~d}, J=7.4 \mathrm{~Hz}, 1 \mathrm{H}), 2.64$ (s, 3H); ${ }^{13} \mathrm{C}$ NMR $\left(75 \mathrm{MHz}, \mathrm{CDCl}_{3}\right): \delta 192.8,140.6,134.2,133.6,132.0,131.7,126.3,19.5$. Physical and spectral data were consistent with those obtained from a commercial sample.

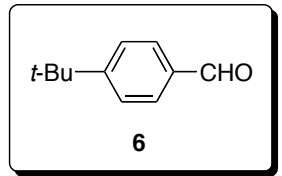

4-tert-Butylbenzaldehyde (Table 2, entry 3): Subjection of 4-tert-butylbenzoyl chloride (5) $(1.0 \mathrm{mmol}, 197 \mathrm{mg})$ to the general procedure afforded after column chromatography (silica gel, $10 \% \mathrm{Et}_{2} \mathrm{O} /$ pentane) $0.161 \mathrm{~g}(99 \%)$ of 4-tert-butylbenzaldehyde (6) as a colorless liquid. ${ }^{1} \mathrm{H}$ NMR $\left(300 \mathrm{MHz}, \mathrm{CDCl}_{3}\right): \delta 9.96(\mathrm{~s}, 1 \mathrm{H})$, $7.81(\mathrm{~d}, J=8.5 \mathrm{~Hz}, 2 \mathrm{H}), 7.54(\mathrm{~d}, J=8.2 \mathrm{~Hz}, 2 \mathrm{H}), 1.32(\mathrm{~s}, 9 \mathrm{H}) ;{ }^{13} \mathrm{C}$ NMR $\left(75 \mathrm{MHz}, \mathrm{CDCl}_{3}\right): \delta$ $191.9,158.4$, 134.1, 129.6, 125.9, 35.3, 31.0. Physical and spectral data were consistent with those obtained from a commercial sample.

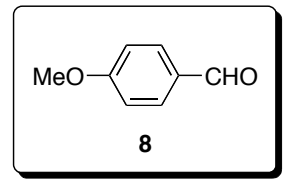

4-Methoxybenzaldehyde (Table 2, entry 4): Subjection of 4-methoxybenzoyl chloride $(7)(1.0 \mathrm{mmol}, 0.171 \mathrm{mg})$ to the general procedure afforded after column chromatography (silica gel, $10 \% \mathrm{Et}_{2} \mathrm{O} /$ pentane) $0.124 \mathrm{~g}(91 \%)$ of 4-methoxybenzaldehyde (8) as a colorless liquid. ${ }^{1} \mathrm{H}$ NMR $\left(300 \mathrm{MHz}, \mathrm{CDCl}_{3}\right): \delta 9.84(\mathrm{~s}, 1 \mathrm{H}), 7.81$ $(\mathrm{d}, J=8.8 \mathrm{~Hz}, 2 \mathrm{H}), 6.97(\mathrm{~d}, J=8.8 \mathrm{~Hz}, 2 \mathrm{H}), 3.84(\mathrm{~s}, 3 \mathrm{H}) ;{ }^{13} \mathrm{C} \mathrm{NMR}\left(75 \mathrm{MHz}, \mathrm{CDCl}_{3}\right): \delta 190.7$, 164.6, 131.9, 129.9, 114.3, 55.5. Physical and spectral data were consistent with those obtained from a commercial sample. 


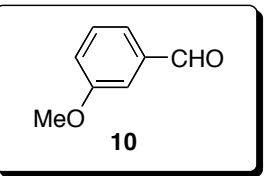

3-Methoxybenzaldehyde (Table 2, entry 5): Subjection of 3-methoxybenzoyl chloride $(9)(1.0 \mathrm{mmol}, 0.171 \mathrm{mg})$ to the general procedure afforded after column chromatography (silica gel, $10 \% \mathrm{Et}_{2} \mathrm{O} /$ pentane) $0.133 \mathrm{~g}(98 \%)$ of 3-methoxybenzaldehyde (10) as a colorless liquid. ${ }^{1} \mathrm{H}$ NMR $\left(300 \mathrm{MHz}, \mathrm{CDCl}_{3}\right): \delta 9.92(\mathrm{~s}, 1 \mathrm{H})$, $7.40(\mathrm{~m}, 2 \mathrm{H}), 7.33(\mathrm{~s}, 1 \mathrm{H}), 7.14(\mathrm{~m}, 1 \mathrm{H}), 3.81(\mathrm{~s}, 3 \mathrm{H}) ;{ }^{13} \mathrm{C}$ NMR $\left(75 \mathrm{MHz}, \mathrm{CDCl}_{3}\right): \delta$ 192.0, 160.1, 137.7, 129.9, 123.4, 121.4, 112.0, 55.3. Physical and spectral data were consistent with those obtained from a commercial sample.

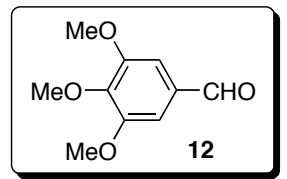

3,4,5-trimethoxybenzaldehyde (Table 2, entry 6): Subjection of 3,4,5trimethoxybenzoyl chloride (11) $(1.0 \mathrm{mmol}, 231 \mathrm{mg})$ to the general procedure afforded after column chromatography (silica gel, $10 \% \mathrm{Et}_{2} \mathrm{O} /$ pentane) $0.160 \mathrm{~g}$ (82\%) of 3,4,5-trimethoxybenzaldehyde (12) as white crystals. ${ }^{1} \mathrm{H} \mathrm{NMR}\left(300 \mathrm{MHz}, \mathrm{CDCl}_{3}\right): \delta 9.84$ (s, 1H), 7.01 (s, 2H), 3.91 (s, 3H), $3.90(\mathrm{~s}, 6 \mathrm{H},) ;{ }^{13} \mathrm{C}$ NMR $\left(75 \mathrm{MHz}, \mathrm{CDCl}_{3}\right): \delta$ 191.0, 153.6, 143.7, $131.7,106.7,61.0,56.3 ; \mathrm{mp} 71-72{ }^{\circ} \mathrm{C}$. Physical and spectral data were consistent with those obtained from a commercial sample.

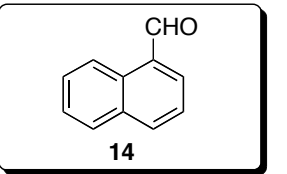

1-Naphthaldehyde (Table 2, entry 7): Subjection of 1-naphthoyl chloride (13) $(1.0 \mathrm{mmol}, 0.191 \mathrm{mg})$ to the general procedure afforded after column chromatography (silica gel, $10 \% \mathrm{Et}_{2} \mathrm{O} /$ pentane) $0.134 \mathrm{~g}(86 \%)$ of 1 naphthaldehyde (14) as a colorless liquid. ${ }^{1} \mathrm{H}$ NMR (300 MHz, $\left.\mathrm{CDCl}_{3}\right): \delta 10.36(\mathrm{~s}, 1 \mathrm{H}), 9.25$ (d, $J$ $=8.5 \mathrm{~Hz}, 1 \mathrm{H}), 8.06(\mathrm{~d}, J=8.2 \mathrm{~Hz}, 1 \mathrm{H}), 7.95(\mathrm{~d}, J=8.2 \mathrm{~Hz}, 1 \mathrm{H}), 7.90(\mathrm{~d}, J=8.5 \mathrm{~Hz}, 1 \mathrm{H}), 7.67(\mathrm{t}, J$ $=8.5 \mathrm{~Hz}, 1 \mathrm{H}), 7.59(\mathrm{~m}, 2 \mathrm{H}) ;{ }^{13} \mathrm{C}$ NMR $\left(75 \mathrm{MHz}, \mathrm{CDCl}_{3}\right): \delta 193.3,136.5,135.1,133.6,131.3$, $130.4,128.9,128.4,126.8,124.7$ (2C). Physical and spectral data were consistent with those obtained from a commercial sample.

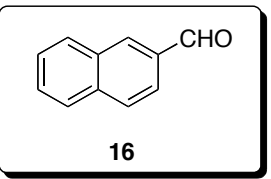

2-Naphthaldehyde (Table 2, entry 8): Subjection of 2-naphthoyl chloride (15) $(1.0 \mathrm{mmol}, 0.191 \mathrm{mg})$ to the general procedure afforded after column chromatography (silica gel, $10 \% \mathrm{Et}_{2} \mathrm{O} /$ pentane) $0.126 \mathrm{~g}(81 \%)$ of 2 naphthaldehyde (16) as white crystals. ${ }^{1} \mathrm{H}$ NMR $\left(300 \mathrm{MHz}, \mathrm{CDCl}_{3}\right): \delta 10.12(\mathrm{~s}, 1 \mathrm{H}), 8.30(\mathrm{~s}, 1 \mathrm{H})$, 7.95-7.85 (m, 4H), 7.63-7.52 (m, 2H); ${ }^{13} \mathrm{C}$ NMR (75 MHz, $\left.\mathrm{CDCl}_{3}\right): \delta 191.8,136.1,134.1,133.7$, 132.3, 129.1, 128.7 (2C), 127.7, 126.7, 122.4; mp 57-58 ${ }^{\circ} \mathrm{C}$. Physical and spectral data were consistent with those obtained from a commercial sample.

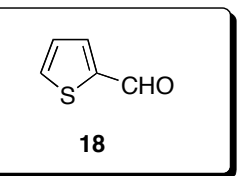

2-Thiophenecarboxaldehyde (Table 2, entry 9): Subjection of 2thiophenecarbonyl chloride (17) $(1.0 \mathrm{mmol}, 0.147 \mathrm{mg})$ to the general procedure afforded after column chromatography (silica gel, 10\% $\mathrm{Et}_{2} \mathrm{O} /$ pentane) $0.103 \mathrm{~g}(92 \%)$ of 2-thiophenecarboxaldehyde (18) as a colorless liquid. ${ }^{1} \mathrm{H}$ NMR 
(300 MHz, CDCl $): \delta 9.90(\mathrm{~s}, 1 \mathrm{H}), 7.75(\mathrm{~m}, 2 \mathrm{H}), 7.19(\mathrm{~m}, 1 \mathrm{H}) ;{ }^{13} \mathrm{C} \mathrm{NMR}\left(75 \mathrm{MHz}, \mathrm{CDCl}_{3}\right): \delta 182.9$, 144.0, 136.2, 135.0, 128.2. Physical and spectral data were consistent with those obtained from a commercial sample.

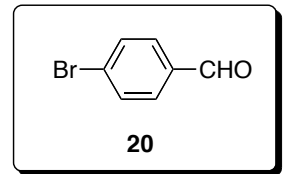

4-Bromobenzaldehyde (Table 2, entry 10): Subjection of 4-bromo-benzoyl chloride (19) $(1.0 \mathrm{mmol}, 220 \mathrm{mg})$ to the general procedure afforded after column chromatography (silica gel, $10 \% \mathrm{Et}_{2} \mathrm{O} /$ pentane) $0.126 \mathrm{~g}(68 \%)$ of 4 bromobenzaldehyde (20) as white crystals. ${ }^{1} \mathrm{H}$ NMR $\left(300 \mathrm{MHz}, \mathrm{CDCl}_{3}\right): \delta 9.93(\mathrm{~s}, 1 \mathrm{H}), 7.72(\mathrm{~d}, J$ $=8.5 \mathrm{~Hz}, 2 \mathrm{H}), 7.65(\mathrm{~d}, J=8.5 \mathrm{~Hz}, 2 \mathrm{H}) ;{ }^{13} \mathrm{C} \operatorname{NMR}\left(75 \mathrm{MHz}, \mathrm{CDCl}_{3}\right): \delta 190.9,135.0,132.4,130.9$, 129.7; mp 54-56 ${ }^{\circ} \mathrm{C}$. Physical and spectral data were consistent with those obtained from a commercial sample. 


$$
1
$$




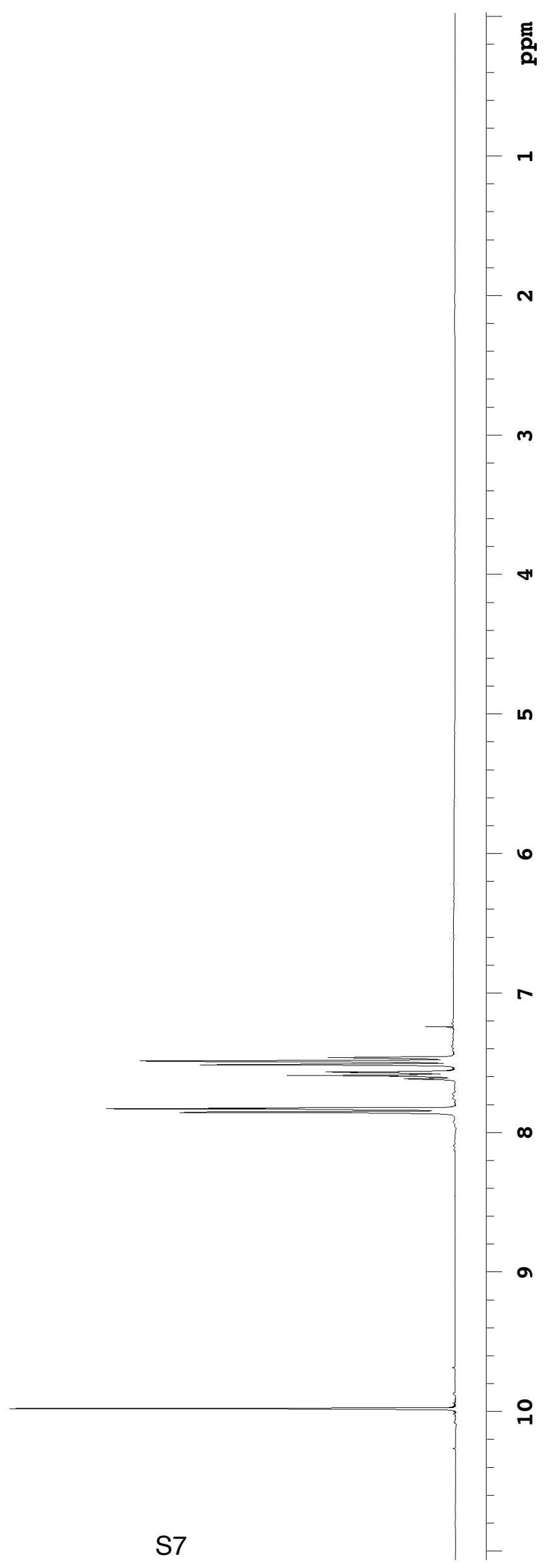




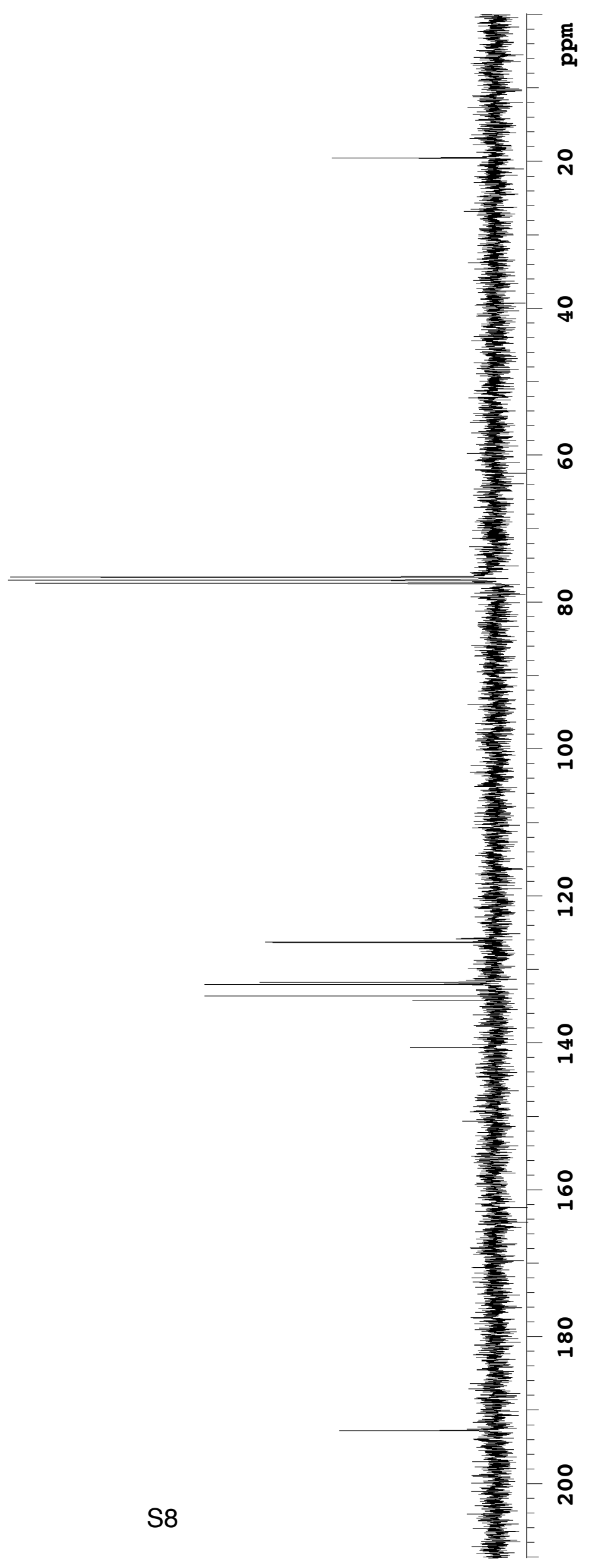




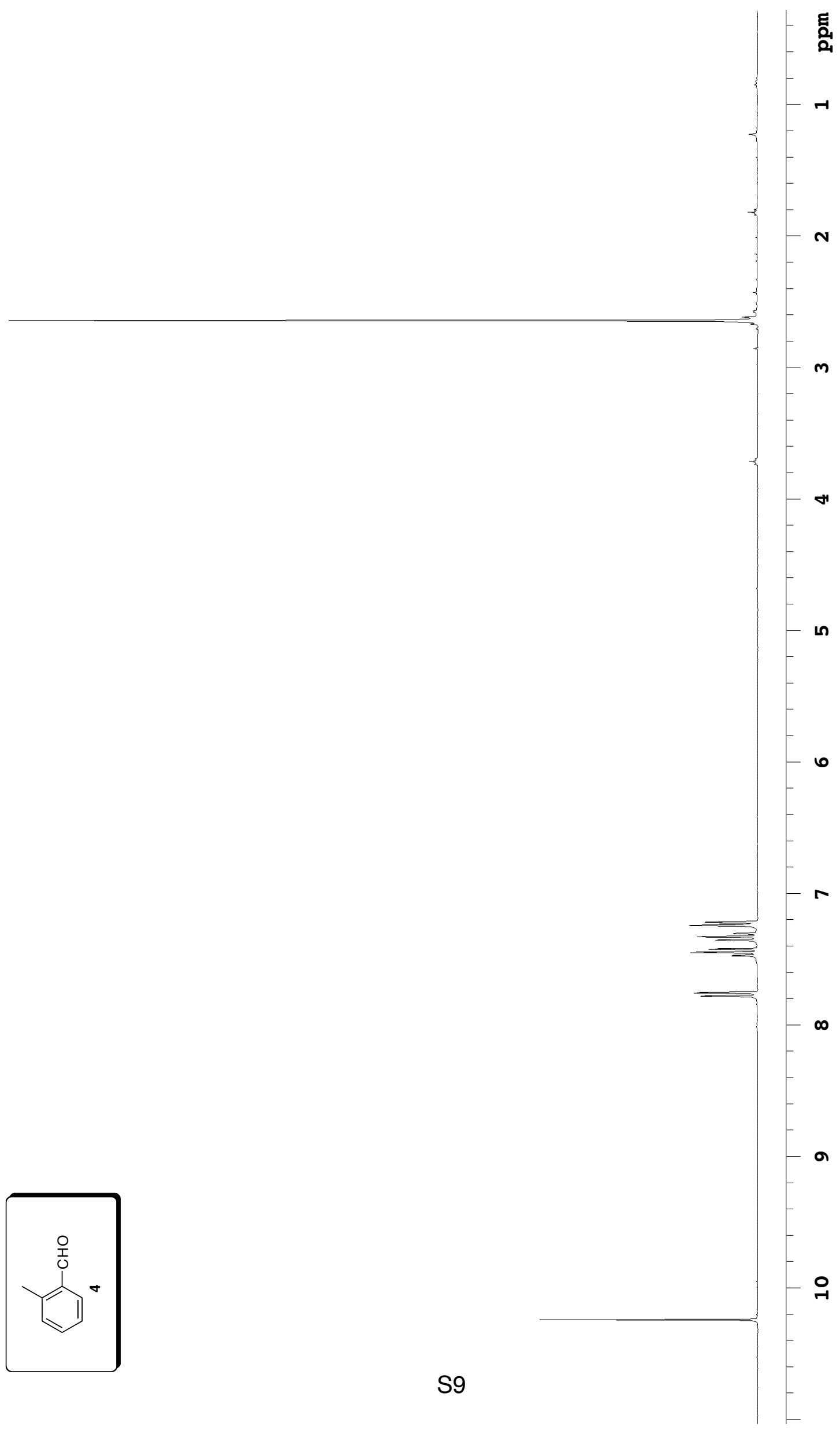




$$
1
$$




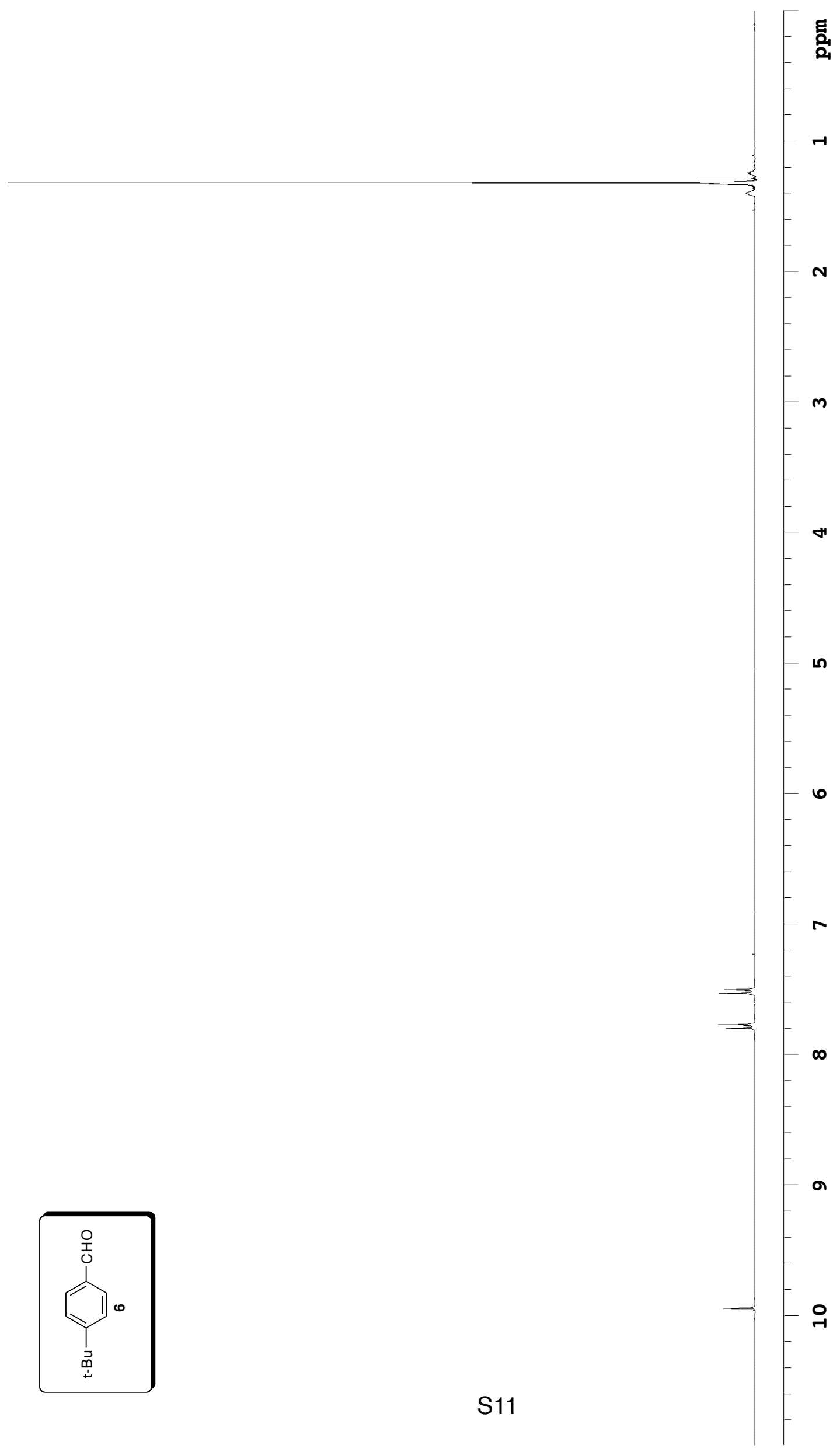




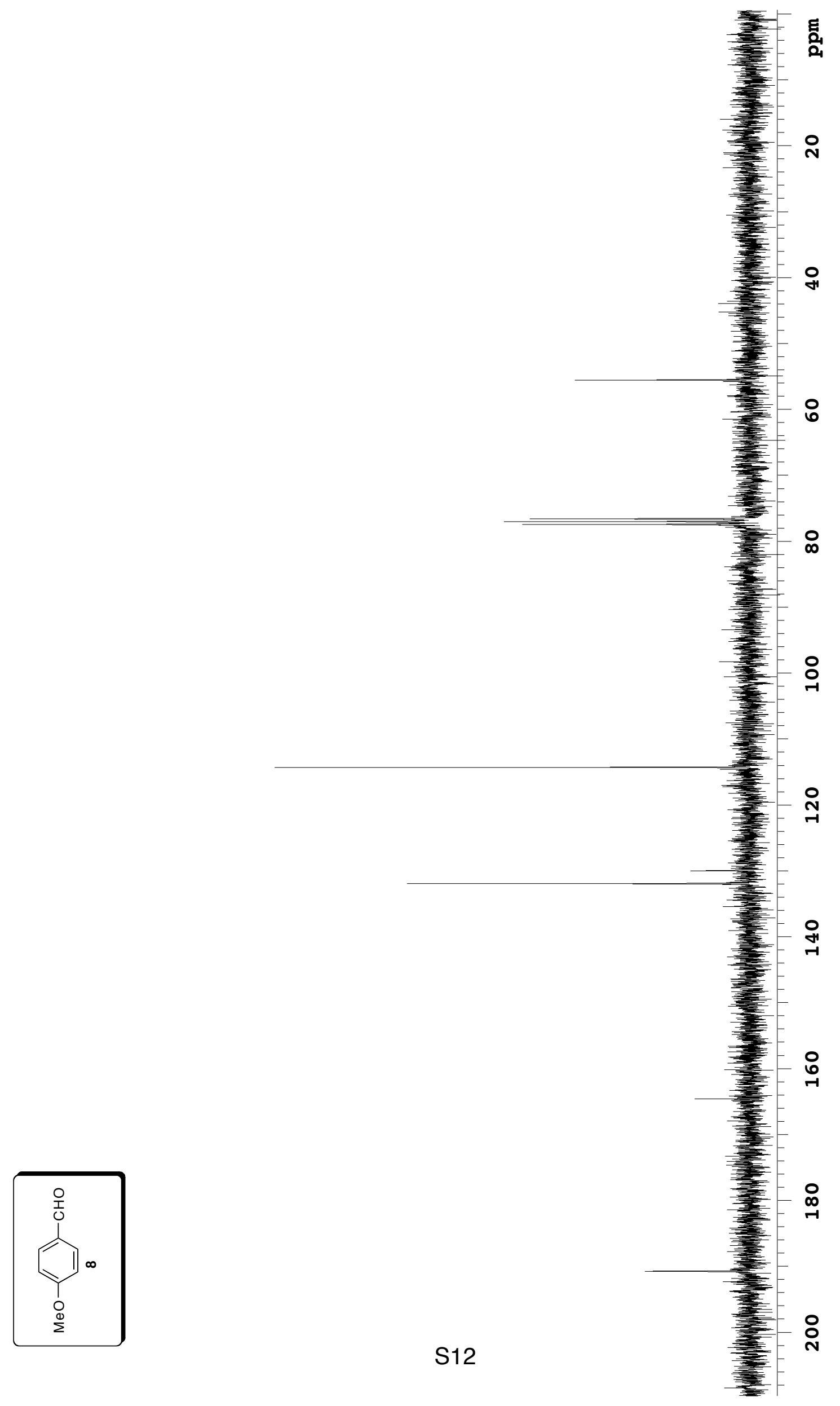




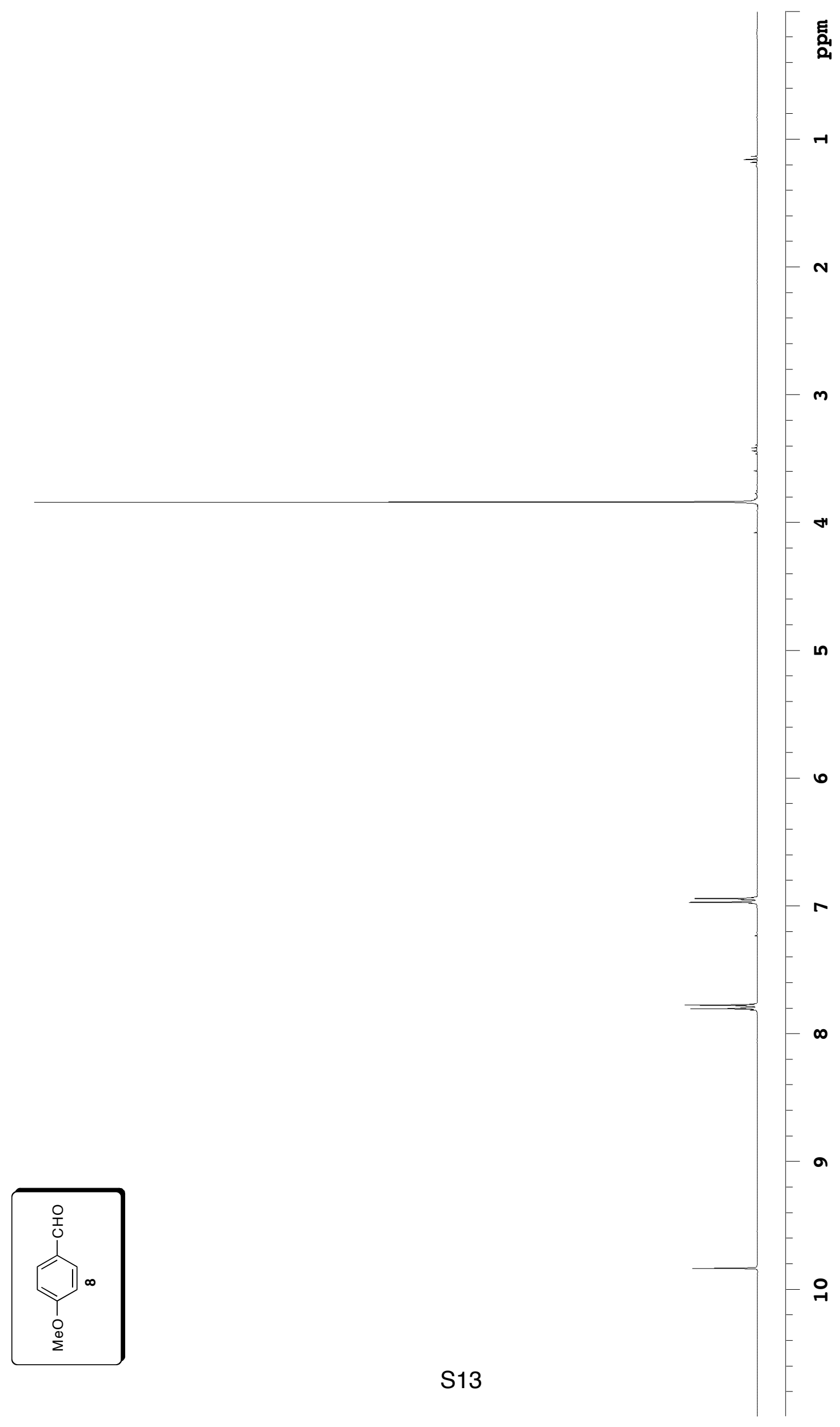




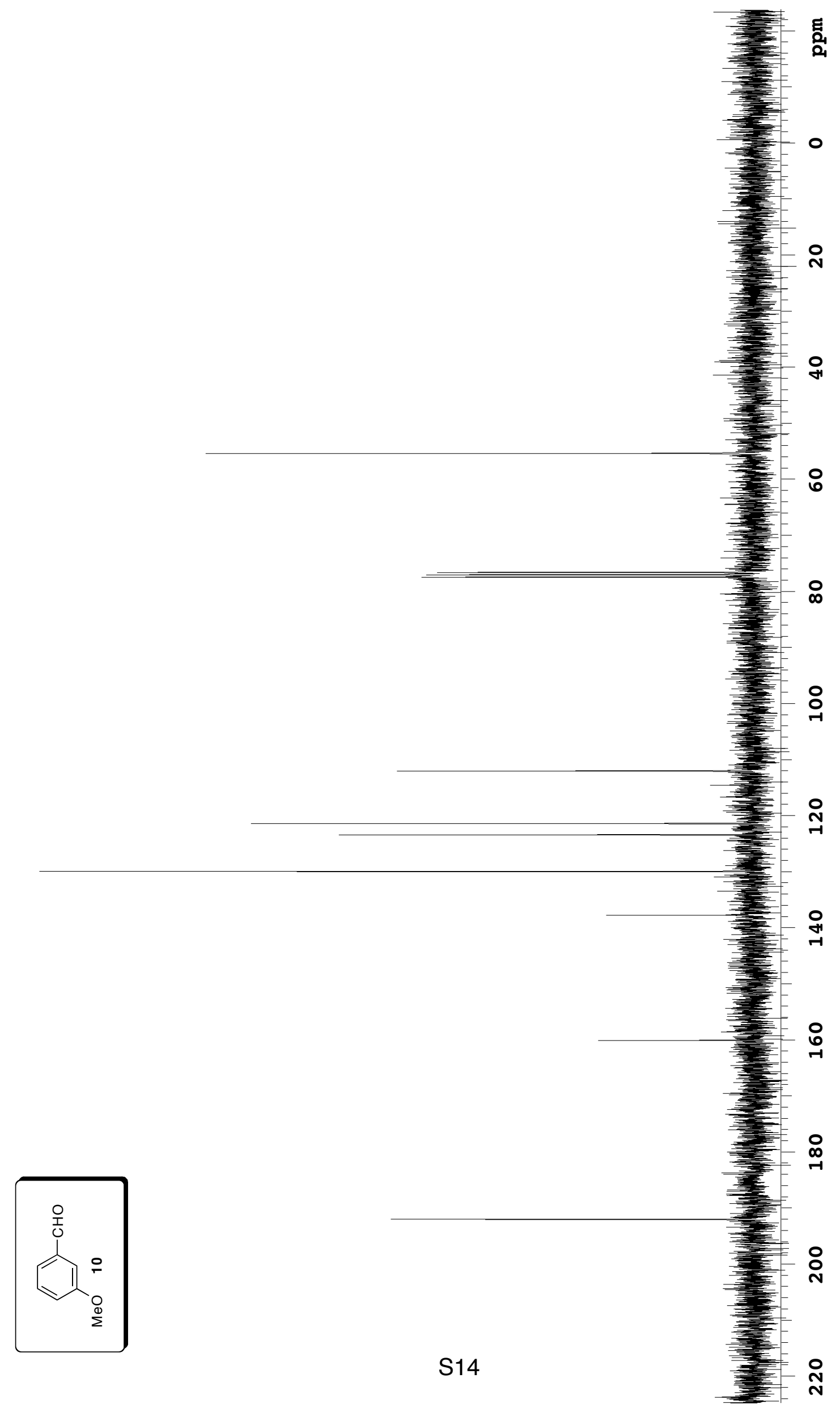




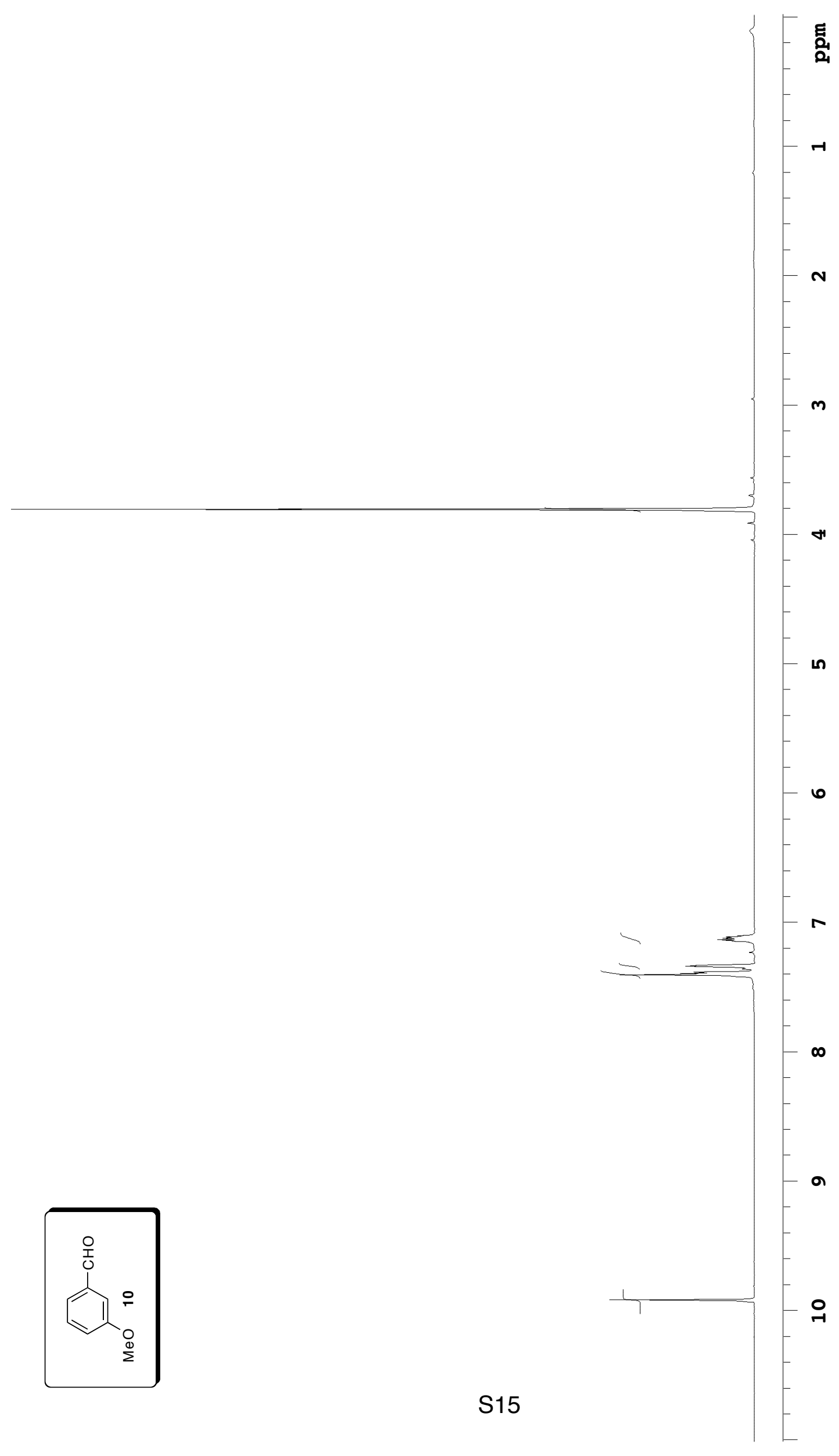




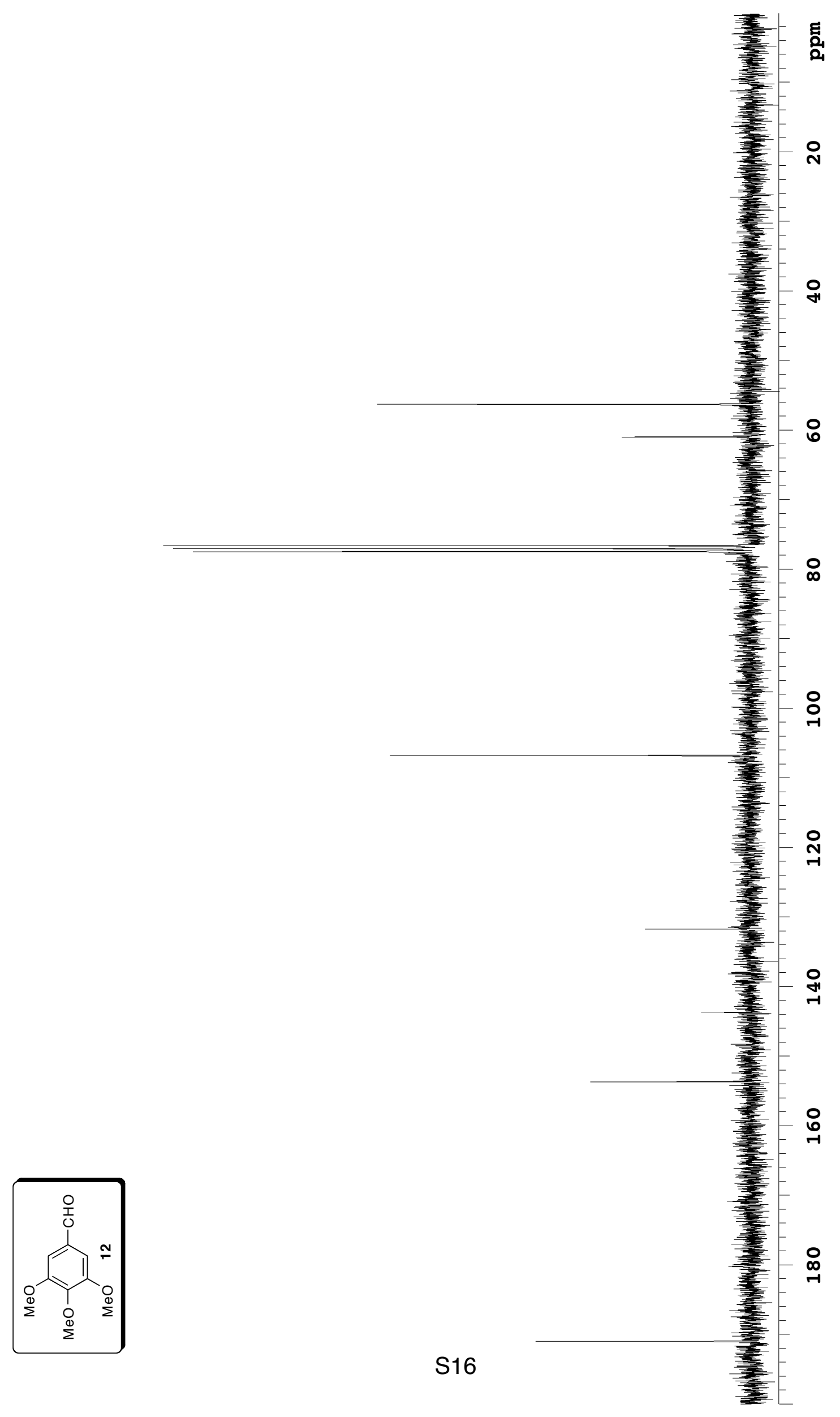




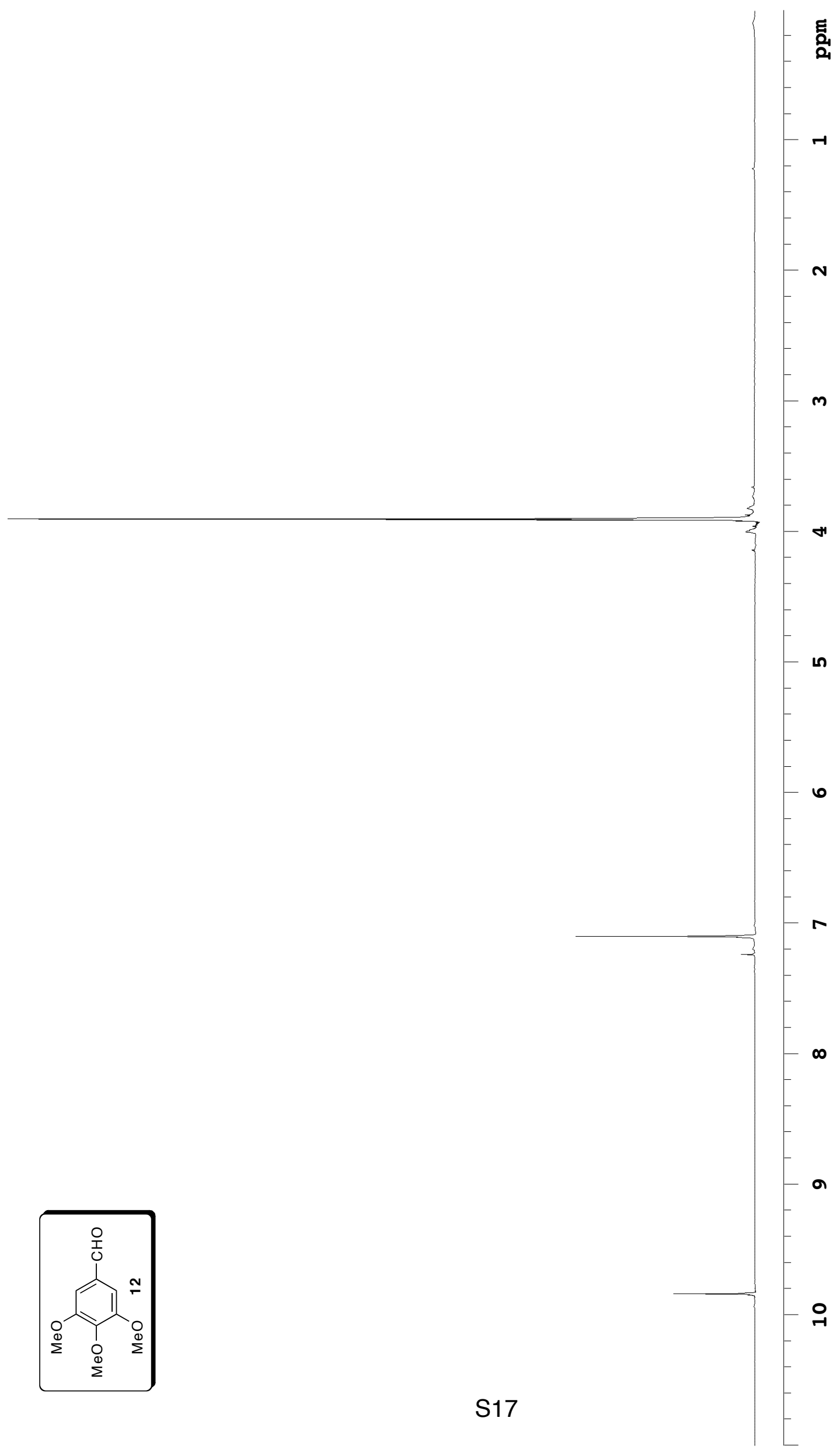




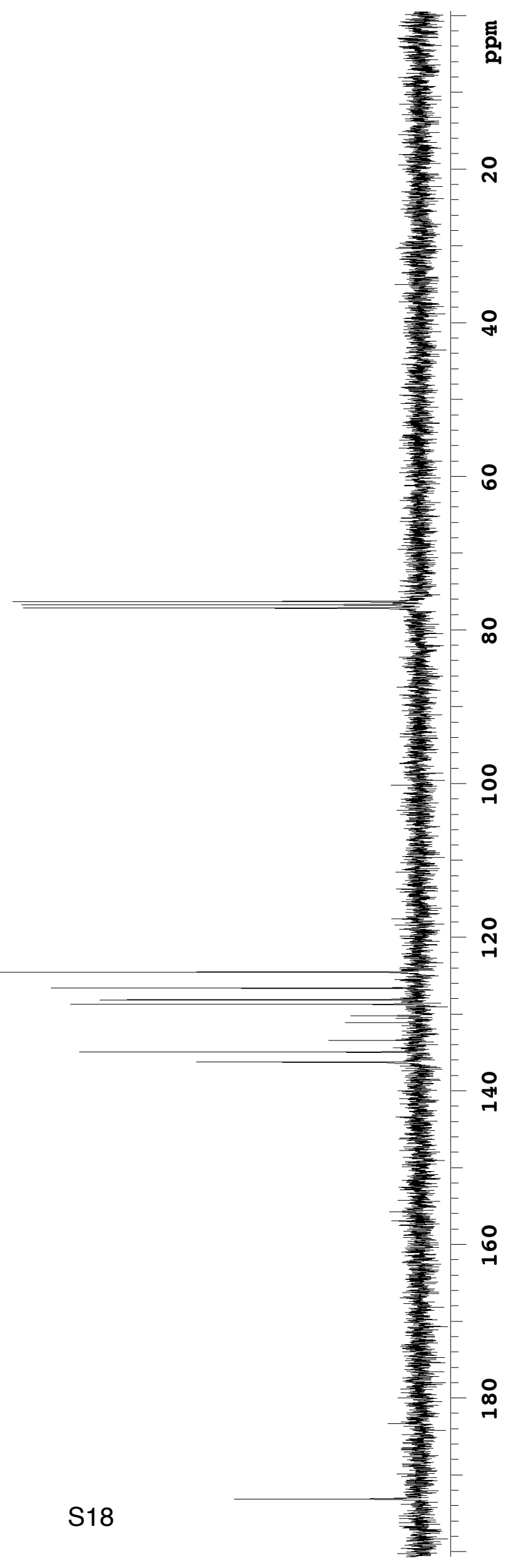




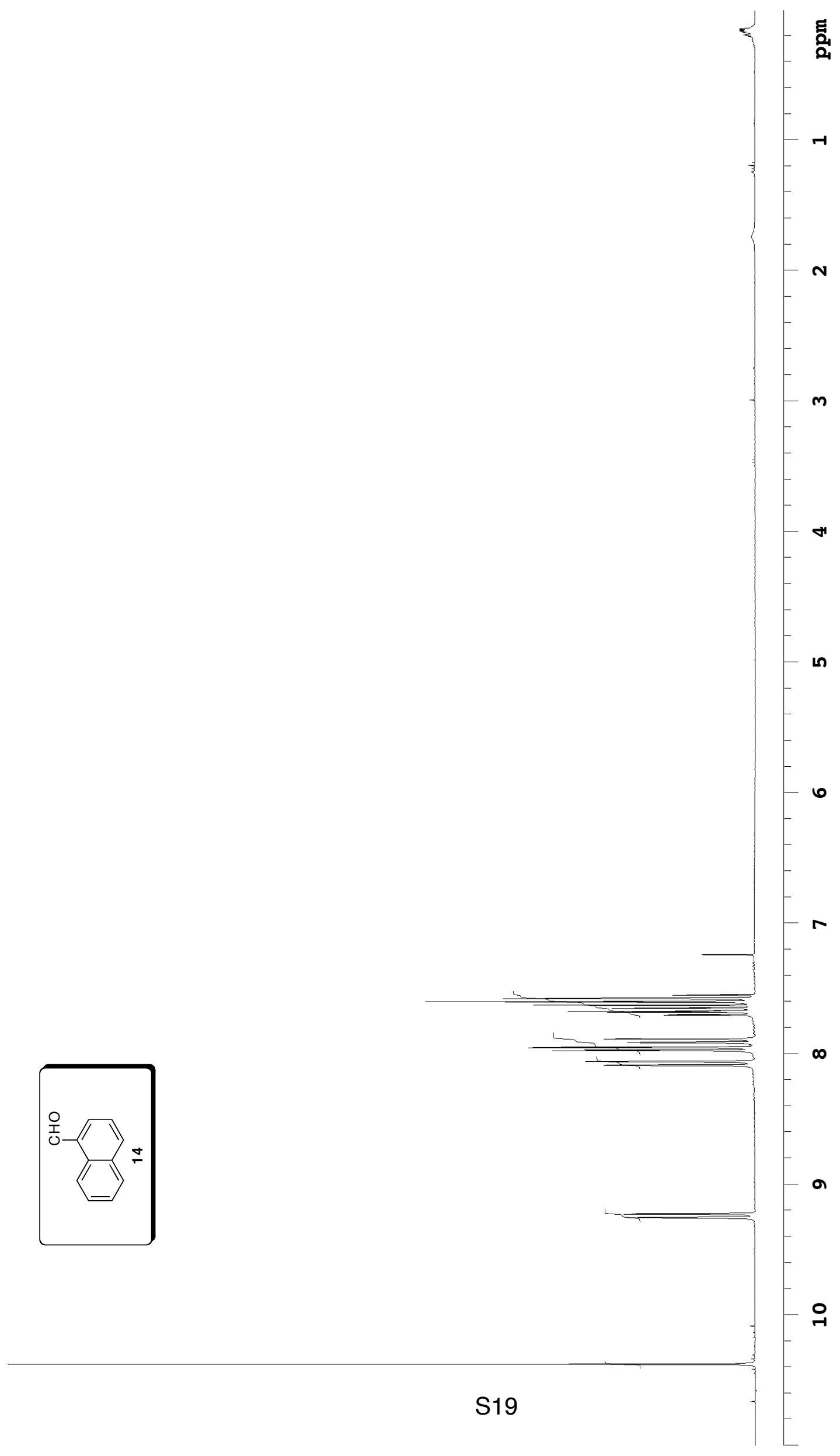




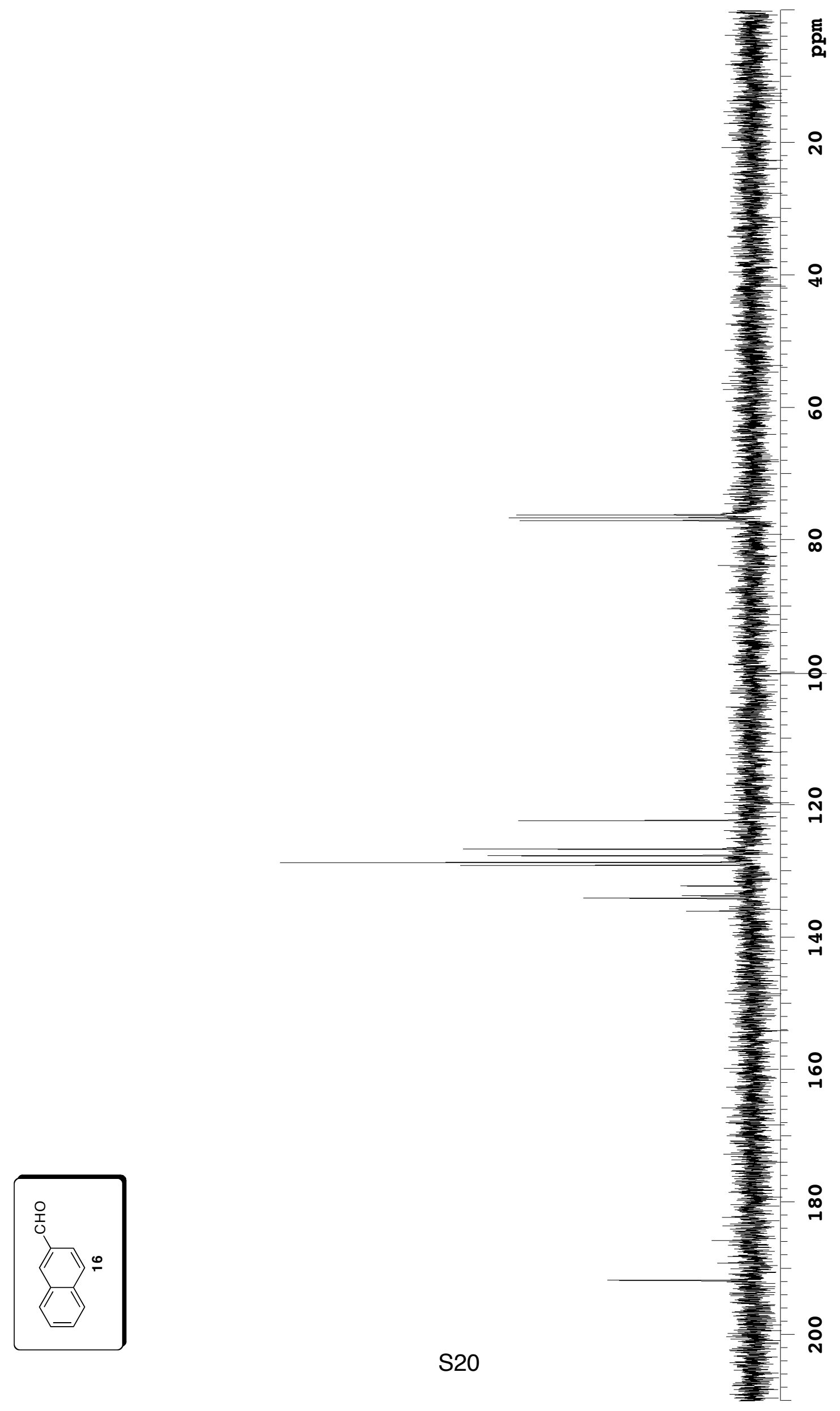




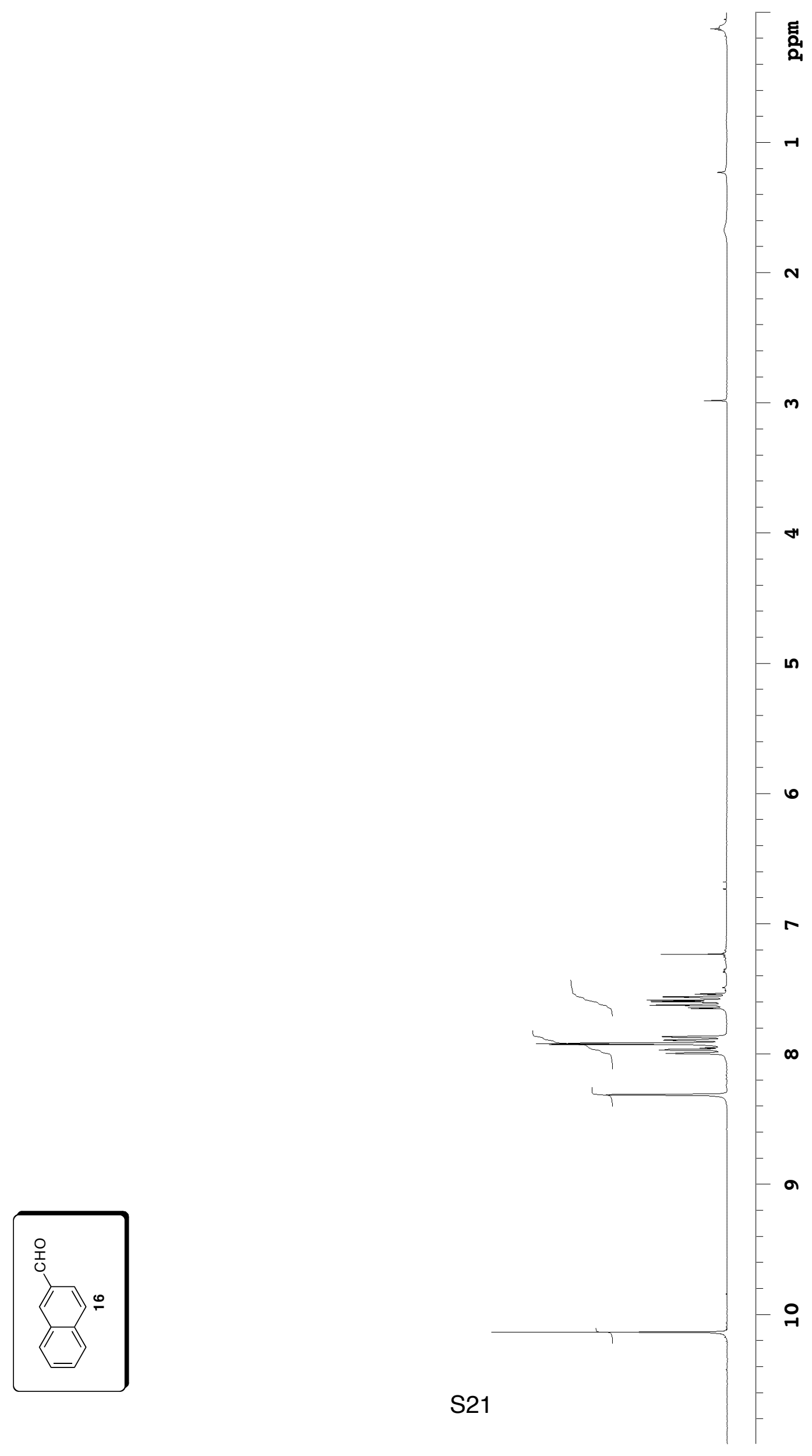




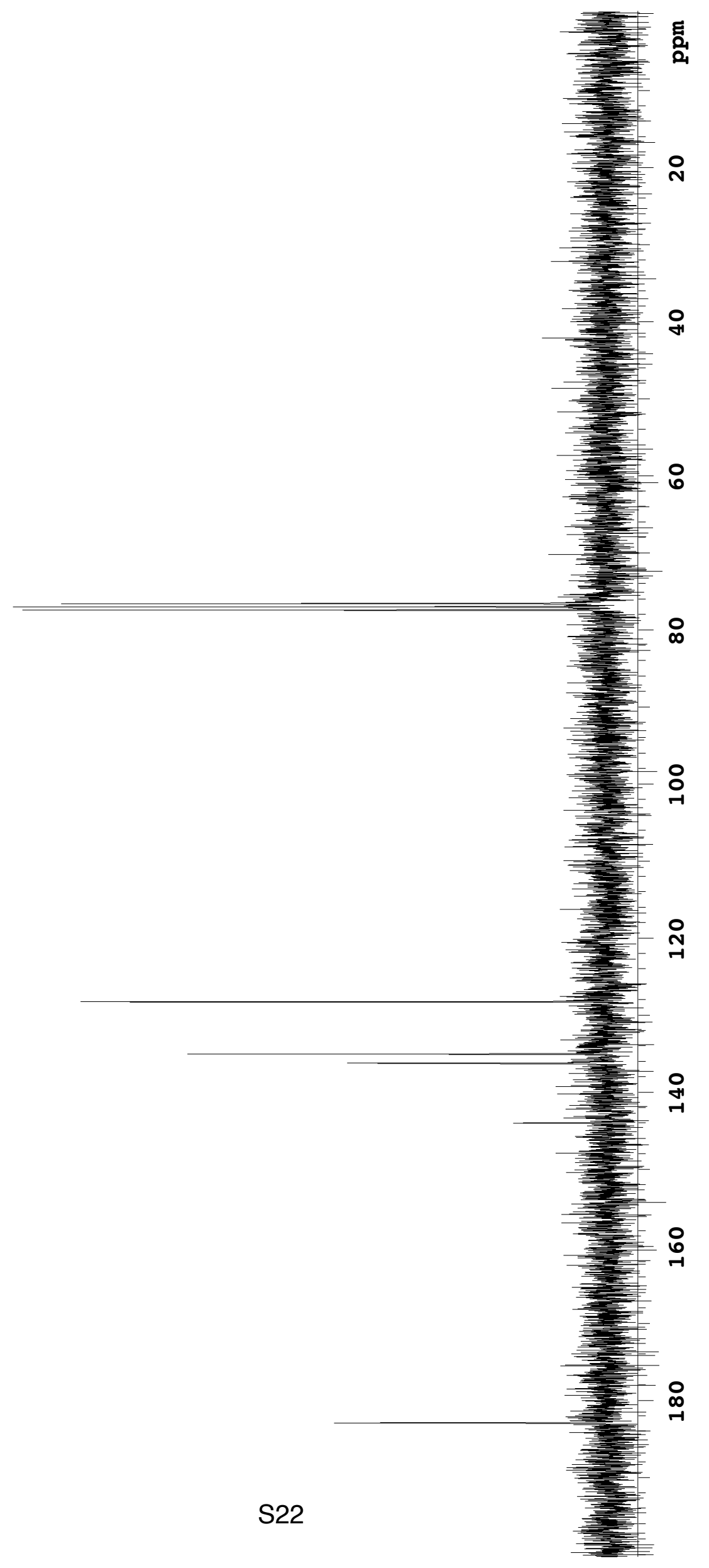




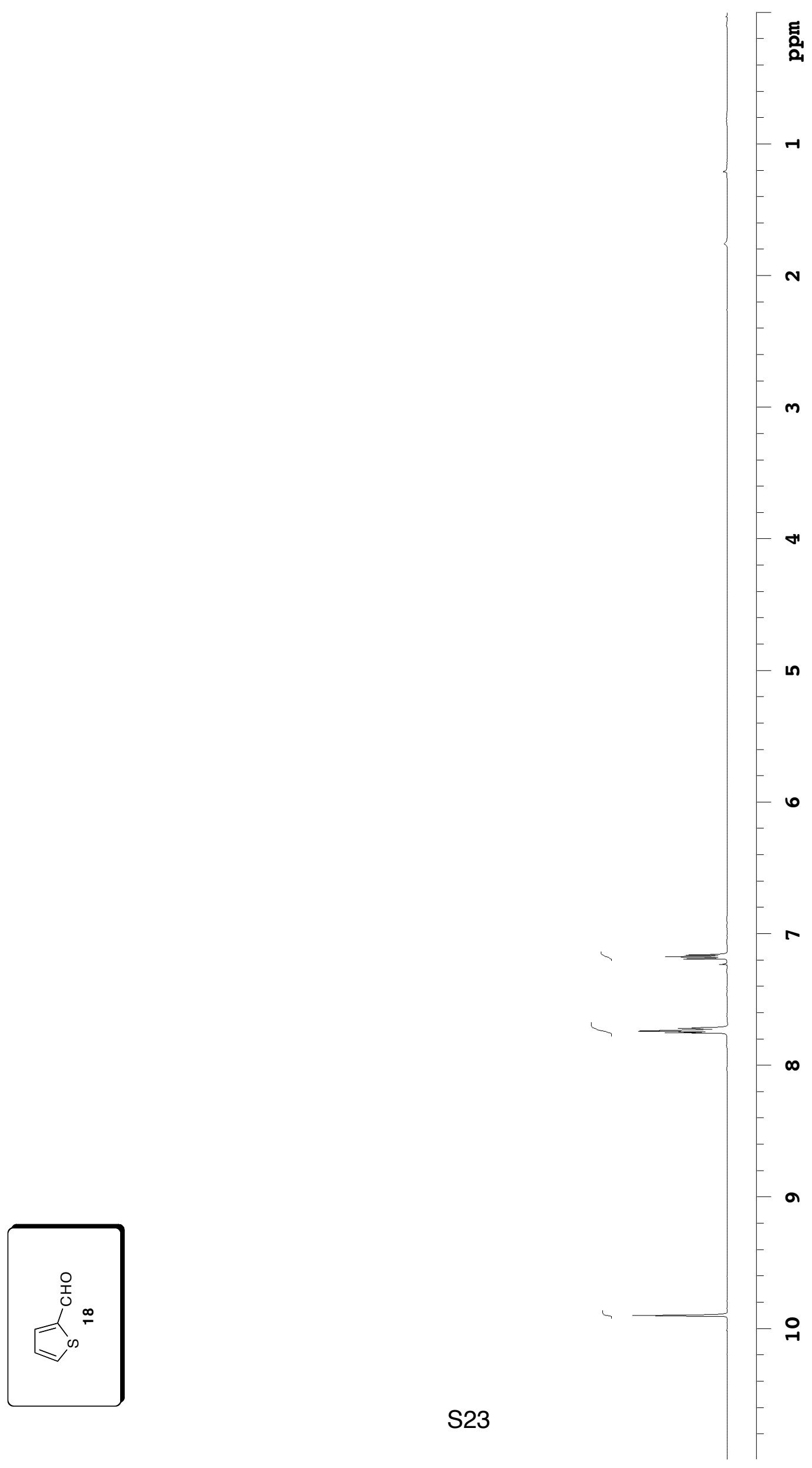




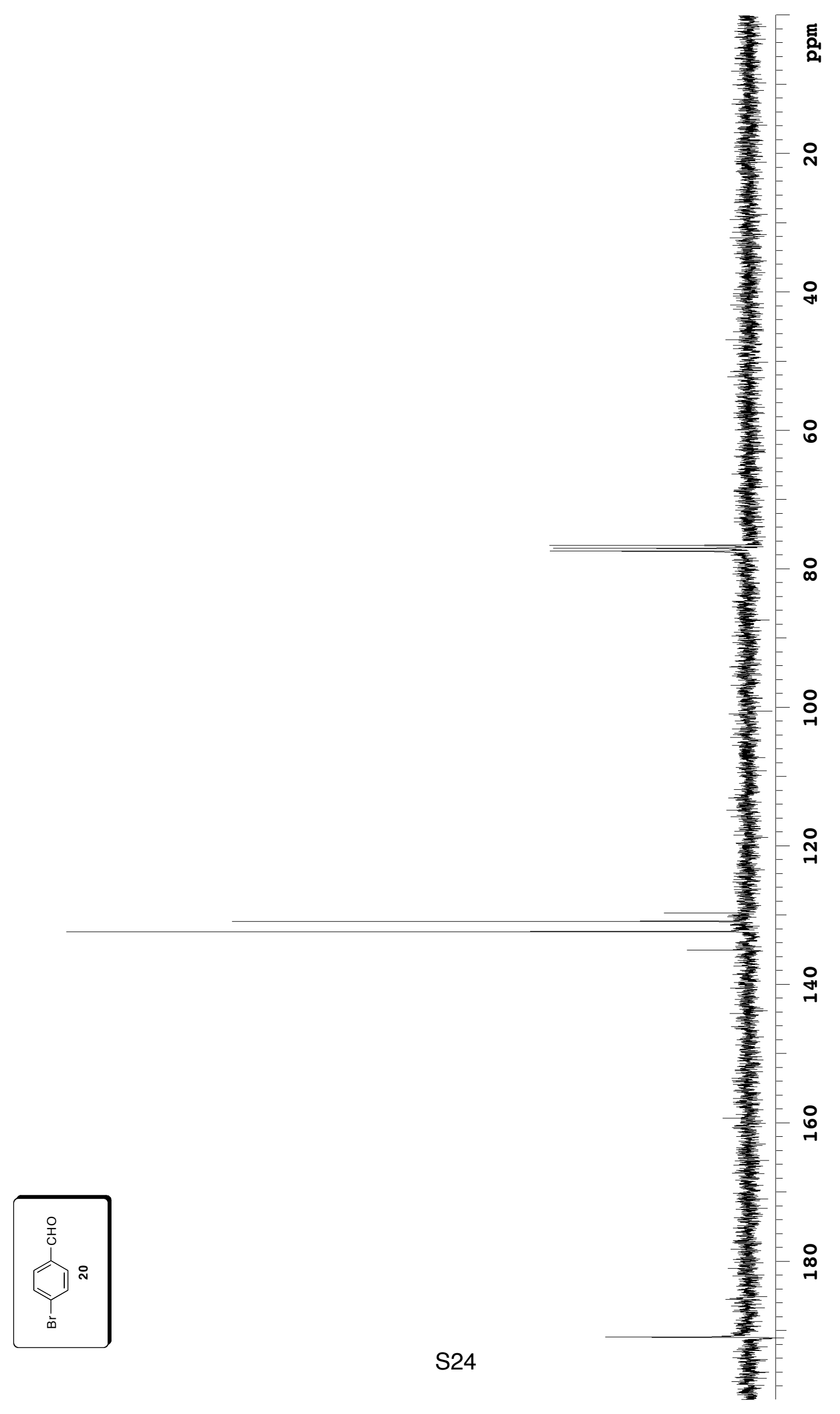




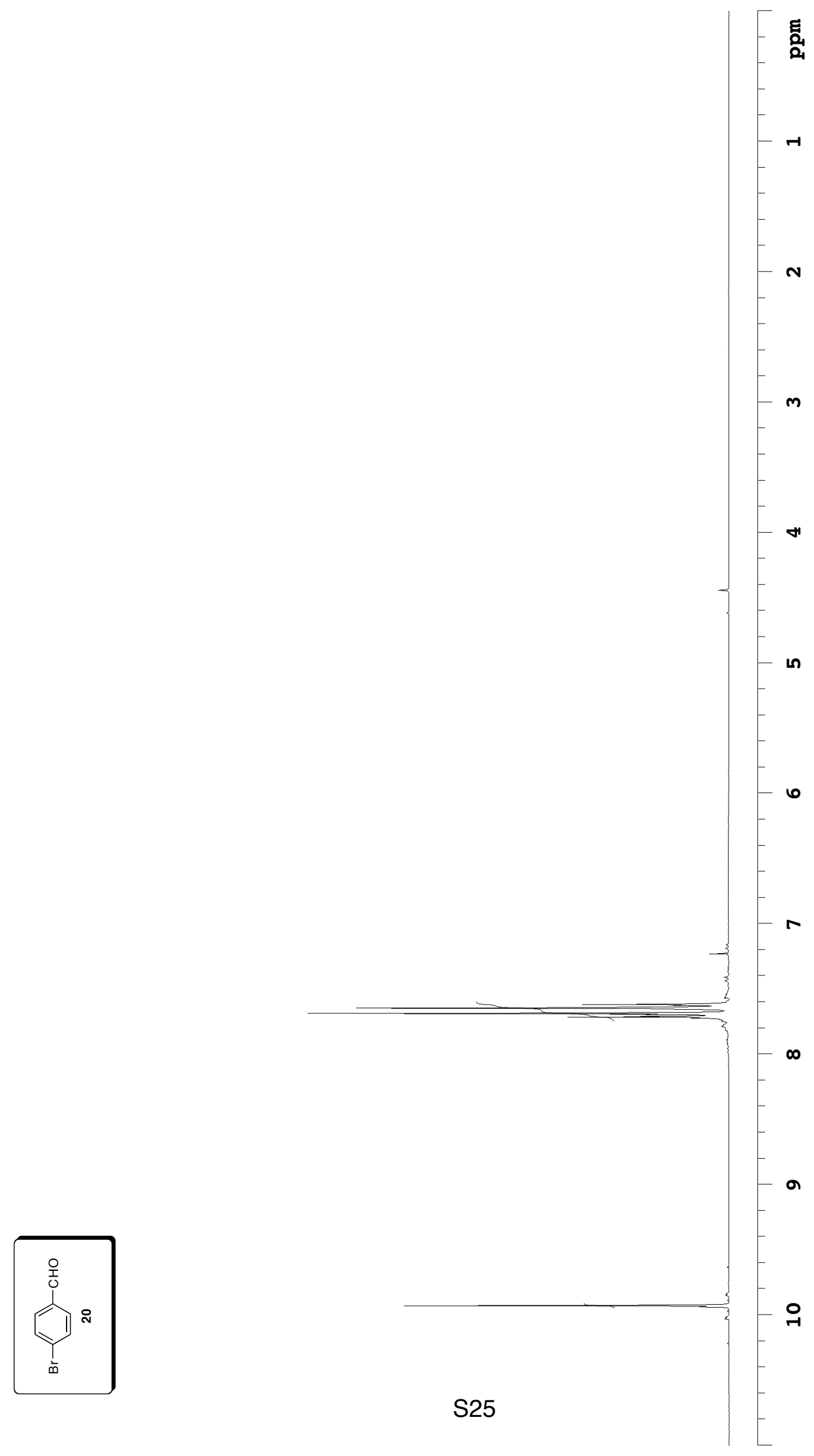

